Revue d'histoire de l'Amérique française

REVUE D.HISTOIRE DE L'AMÉRIQUE FRANÇAISE

\title{
Conscience coloniale et conscience internationale dans les écrits publics de Louis-Joseph Papineau (1815-1839)
}

\section{Yvan Lamonde}

Volume 51, numéro 1, été 1997

URI : https://id.erudit.org/iderudit/305621ar

DOI : https://doi.org/10.7202/305621ar

Aller au sommaire du numéro

Éditeur(s)

Institut d'histoire de l'Amérique française

ISSN

0035-2357 (imprimé)

1492-1383 (numérique)

Découvrir la revue

Citer cet article

Lamonde, Y. (1997). Conscience coloniale et conscience internationale dans les écrits publics de Louis-Joseph Papineau (1815-1839). Revue d'histoire de l'Amérique française, 51(1), 3-37. https://doi.org/10.7202/305621ar

\section{Résumé de l'article}

Le présent article entend répondre à deux questions : quelle conscience Papineau eut-il de la condition coloniale du Bas-Canada et quelle référence a-t-il faite à l'expérience d'autres colonies ou pays dominés en Europe et dans les Amériques avant les rébellions de 1837-1838? La réponse à ces questions permet de renouveler la connaissance historique sur Papineau, sur son " patriotisme ", sur son passage du britannisme au républicanisme, sur sa francophilie toute relative et sa conscience aiguë d'appartenance à l'Amérique. S'il faut expliquer l'absence de référence à l'expérience émancipatrice contemporaine de la Grèce, de l'Italie ou de la Pologne, il convient d'observer que la stratégie de Papineau consiste à trouver une diagonale entre l'expérience réussie des anciennes colonies devenues les États-Unis et celle en voie de réussite possible de l'Irlande. 


\title{
CONSCIENCE COLONIALE ET CONSCIENCE INTERNATIONALE DANS LES ÉCRITS PUBLICS DE LOUIS-JOSEPH PAPINEAU (1815-1839)
}

\author{
YVAN LAMONDE \\ Département de langue et littérature françaises \\ Université McGill*
}

\section{RÉSUMÉ}

Le présent article entend répondre à deux questions: quelle conscience Papineau eut-il de la condition coloniale du Bas-Canada et quelle référence a-t-il faite à l'expérience d'autres colonies ou pays dominés en Europe et dans les Amériques avant les rébellions de 1837-1838? La réponse à ces questions permet de renouveler la connaissance historique sur Papineau, sur son «patriotisme», sur son passage du britannisme au républicanisme, sur sa francophilie toute relative et sa conscience aiguë d'appartenance à l'Amérique. S'il faut expliquer l'absence de référence à l'expérience émancipatrice contemporaine de la Grèce, de l'Italie ou de la Pologne, il convient d'observer que la stratégie de Papineau consiste à trouver une diagonale entre l'expérience réussie des anciennes colonies devenues les États-Unis et celle en voie de réussite possible de l'Irlande.

\section{ABSTRACT}

The following article intends to answer these two questions: to which extent was Papineau conscious of the colonial condition of Lower Canada and which reference did he make, before the 1837-1838's rebellions, to the experience of other colonies or dominated countries in Europe and in the Americas? The answer to these questions renews our knowledge of Papineau, of his "patriotism», of his transition from britannism to republicanism, of his quite relative francophilia and his sharp consciousness of a belonging to America. If the absence of reference made to the contemporaneous emancipatory experiences of Greece, Italy or Poland must be explained, it is clear that Papineau's strategy consists in finding something like a midway between the successful experience of the Thirteen colonies and the conceivable one of Ireland.

* Pour leurs commentaires sur ce texte, je remercie Jean-Paul Bernard de l'Université du Québec à Montréal et Gérard Bouchard de l'Institut interuniversitaire de recherches sur les populations, dans le cadre duquel sont menées ces recherches sur Papineau et sur la référence aux mouvements nationalitaires européens et américains au Bas-Canada de 1808 à 1838. De l'évaluateur «B» de mon texte pour la revue - qui ne se connaît pas comme tel mais qui se reconnaîtra - j'ai retenu une interprétation particulièrement originale de la non-référence chez Papineau à certaines expériences nationales. 
Cette étude sur Papineau s'inscrit dans le cadre général d'une recherche sur la référence aux mouvements nationalitaires européens et américains faite au Bas-Canada entre 1808 et 1838, c'est-à-dire entre l'amorce des mouvements d'indépendance coloniale en Amérique latine et l'entrée de Papineau en politique (1809), d'une part, et les insurrections de 1837 et de 1838, d'autre part. Cette recherche entend répondre aux trois questions suivantes: quelle connaissance avait-on au Bas-Canada de ces mouvements patriotiques et nationalitaires européens et latino-américains avant les rébellions? Quel usage éditorial et politique a-t-on fait de cette information? Et, plus globalement, quelle référence a-t-on faite au Bas-Canada, avant 1838 et autour de 1848 , au principe des nationalités, à ce que le $\mathrm{XX}^{\mathrm{e}}$ siècle a appelé le droit des peuples à disposer d'eux-mêmes?

Pour répondre à ces questions, nous explorerons huit types de documents: la presse francophone du Bas-Canada; les écrits de quelques journalistes, hommes politiques et écrivains (Garneau, Viger, Parent, Dessaulles, Gonzalve Doutre, Papineau); des écrits des Patriotes (92 Résolutions, correspondances, mémoires et souvenirs); le corpus des textes poétiques de l'époque constitué par Grisé, Lortie et Savard; les récits de voyages (Plessis et Lartigue, Garneau); les mandements des évêques de Québec et de Montréal et divers documents religieux dont la Doctrine de l'Église d'Irlande... de l'abbé Maguire); le corpus des catalogues de bibliothèques et de librairies et finalement le Nouvel abrégé de géographie moderne (1833) de l'abbé Jean Holmes. Dans le cas de Papineau, nous avons, avec la collaboration de Claude Larin, établi la bibliographie - à paraître - de ses textes imprimés et de ses interventions parlementaires rapportées dans la presse ou dans d'autres types d'imprimés. Nous pensons avoir lu Papineau en totalité, à l'exclusion de la correspondance que nous analyserons éventuellement. Nous préparons enfin, toujours avec Claude Larin, une substantielle anthologie de textes de Papineau.

À notre connaissance, la dimension internationale des rébellions de 1837 et de 1838 n'a pas, jusqu'à maintenant, retenu l'attention des historiens de Papineau, des Patriotes et des rébellions, sauf d'Allan Greer qui, en introduction à son récent ouvrage The Patriots and the People. The Rebellion of 1837 in Rural Lower Canada, situe brièvement la problématique des révoltes paysannes dans un contexte international. Si, dans son article-bilan de la Canadian Historical Review (1995), A. Greer en appelle à la comparaison des rébellions non seulement dans le Haut et le Bas-Canada mais aussi à une comparaison internationale des mouvements d'émancipation coloniale et nationale, les travaux publiés qui se rapprochent le plus de notre problématique 
sont ceux, cités ultérieurement (note 31), qui comparent l'Irlande et le Bas-Canada ou O'Connell et Papineau.

Cette absence de la dimension internationale des mouvements d'indépendance ou d'émancipation nous semble une carence importante dans l'étude des rébellions bas-canadiennes, dans la mesure où celles-ci peuvent difficilement être comprises sans effort comparatif. À l'apogée du mouvement d'éveil des nationalités, il est indispensable de s'interroger sur l'ampleur et la spécificité des rébellions et surtout d'en voir les limites, les succès et les échecs à la lumière des références qui sont faites ou pas à des tentatives similaires dans des colonies sous le contrôle de divers empires.

Nous sommes conscient des limites d'une étude qui n'utilise que les écrits imprimés ou publics de Papineau. Outre le fait que nous devions limiter l'ampleur d'une première approche et que la correspondance privée de Papineau se trouve dans divers fonds d'archives (Viger, Roebuck, Mackenzie, à titre d'exemples) et dans des fonds d'archives de Papineau et de sa famille pour lesquels il n'y a pas encore de concordance de la correspondance, nous croyons valable de scruter le seul discours public du chef patriote, celui qui est tenu pour des auditoires acquis ou opposés, locaux ou étrangers et qui comporte d'évidents effets rhétoriques. Nous avons proposé des moyens pour qu'une concordance des fonds d'archives Papineau soit établie dans les meilleurs délais, ce qui facilitera à tous l'exploration de la correspondance privée selon des perspectives diverses. Par ailleurs, l'accès difficile au texte de Papineau légitime notre choix de le citer avec une certaine abondance, de lui donner la parole, ne serait-ce que pour rappeler que l'histoire est une construction qui montre tout de même quelques-uns de ses matériaux.

Ce que nous appelons «conscience» coloniale et internationale chez Papineau réfère à la connaissance et à la mémoire qu'a le chef patriote, de 1815 à 1839, des défis politiques et constitutionnels du Bas-Canada, des positions des autorités métropolitaine et coloniale, des perceptions qu'il a de l'évolution de l'Amérique et de l'Europe, des combats qu'il mène sur les hustings, dans la presse et en Chambre. Cette conscience, qui n'est pas que connaissance, se construit sur la réflexion, sur l'expression des idées et des sentiments, sur la réplique et dans la polémique et inclut un sens profond de responsabilité civique. Formulée autrement, cette expérience cumulative d'analyse, d'expression et de militantisme équivaut à la conscience historique d'un homme politique exceptionnellement présent sur la scène publique à un moment crucial de l'évolution du Bas-Canada. 
Afin d'évaluer la conscience qu'avait Papineau de la situation coloniale du Bas-Canada et de la conjoncture internationale des mouvements d'émancipation nationale, nous analyserons successivement l'évolution de la représentation de l'Angleterre chez le chef du Parti patriote; la place qu'il fait à l'ancienne mère patrie, la France, dans sa pensée politique; le sens de son admiration pour la république des États-Unis; la référence qu'il fait au Royaume-Uni et aux autres colonies britanniques (Irlande, Jamaïque, Écosse, Malte, Haut-Canada, Nouvelle-Écosse); les références nationalitaires absentes de sa conception politique (Italie, Grèce, Belgique, Pologne, Amérique latine); puis, pour prendre la mesure de sa conscience coloniale, nous préciserons la montée de son impatience et de sa désillusion politique et le lexique «patriotique» qui est le sien. Nous pourrons alors conclure sur la géopolitique qui présida à la conception et à l'action politiques du député, de l'orateur de la Chambre d'assemblée et du chef du Parti canadien puis patriote.

\section{PAPINEAU ET L'ANGLETERRE}

Lorsque Papineau entre à la Chambre d'assemblée en 1809 , il y a été précédé par son père et par une génération d'hommes politiques auxquels il emprunte quelques idées et quelques stratégies. Joseph Papineau, qui siège à la Chambre d'assemblée de 1792 à 1804 puis de 1809 à 1814, s'est familiarisé avec les théoriciens du parlementarisme (Locke, Blackstone) tout autant qu'avec Montesquieu, Voltaire et Rousseau. Il est donc vraisemblable que le père, qui, avec d'autres Canadiens, réclament une Chambre d'assemblée, ait communiqué au fils à la fois les ouvrages et les idées de ces penseurs politiques et surtout une certaine conception du politique, de l'Angleterre et de la constitution anglaise. Le père fait ainsi la transition entre la monarchie absolue héritée du Régime français et la monarchie constitutionnelle octroyée par la constitution de 1791. La monarchie parlementaire fait une place à la souveraineté populaire tandis que sa lecture du Contrat social de Rousseau lui fournit un argumentaire en faveur de la subordination du pouvoir exécutif au pouvoir législatif, de la préséance de la Chambre d'assemblée sur le Conseil législatif'.

1. Richard Chabot, «Papineau, Joseph», Dictionnaire biographique du Canada (SainteFoy, Les Presses de l'Université Laval, 1988), 7: 729-733; Fernand Ouellet, «Joseph Papineau et le régime parlementaire (1791)», Éléments d'histoire sociale du Bas-Canada (Montréal, Hurtubise $\mathrm{HMH}, 1972$ ), 225-232. Pierre Tousignant a remis l'historiographie de la démocratie au Québec sur ses pieds en établissant la participation des Canadiens français à la demande d'une Chambre d'assemblée; P. Tousignant, La genèse et l'avénement de la Constitution de 1791, Ph.D. (Histoire), Université de Montréal, 1971, 447-459. 
Joseph Papineau fut donc de la première génération des parlementaires canadiens-français, de ceux qui ont vu dans l'octroi d'une Chambre d'assemblée et surtout dans le respect du principe de la représentation populaire le pivot par excellence d'une affirmation politique de la majorité canadienne-française. La concession par l'Angleterre du droit de vote et de la représentation parlementaire est à l'origine de l'admiration et du respect voués à la métropole par les Bas-Canadiens de langue française; elle est du coup la cause de la désillusion des Bas-Canadiens de langue anglaise, incommodés de voir se constituer ce qu'ils appellent rapidement une «nation canadienne» et de constater que leur pouvoir politique est inférieur à leur pouvoir économique et social. Cette admiration de l'Angleterre, de la constitution, des «principes anglois», c'est-à-dire «libéraux», traverse les propos et écrits de Pierre-Stanislas Bédard, chef du Parti canadien, de Denis-Benjamin Viger, du journal Le Canadien, du docteur Jacques Labrie ou d'un François Blanchet. Cette admiration est toutefois entamée dans Le Canadien et, en particulier, dans le Mémoire (1814) de Bédard par la critique que des milieux politiques canadiens-français commencent à faire du Conseil législatif, de sa composition essentiellement anglophone, de la dépendance financière de ses membres à l'égard du pouvoir et de sa stratégie de blocage des lois votées par la Chambre. Cette critique mènera le Parti canadien puis le Parti patriote à réclamer, à la fin de la décennie 1820, l'électivité du Conseil législatif, trouvant alors son inspiration et ses modèles politiques dans la république étatsunienne où le Sénat est élu. Mais jusqu'à la fin de la décennie 1820, les Canadiens français, qui avaient misé sur la métropole pour régler les tensions coloniales, avaient vu Londres prendre le parti de l'écoute, différer le projet d'Union de 1822 et, encore en 1828, créer un Comité sur les affaires du Canada ${ }^{2}$.

Dans les années 1820, Louis-Joseph Papineau reste admiratif de la monarchie constitutionnelle anglaise et de la constitution que l'Angleterre s'est donnée en 1688. Quelques mois après le décès de George III, il explique ainsi les transformations positives amenées par la cession de la Nouvelle-France à l'Angleterre:

Rappelons-nous que sous le gouvernement français (arbitraire et oppresseur, à l'intérieur comme au dehors) les intérêts de cette colonie avaient été plus souvent négligés et mal administrés que

2. Yvan Lamonde «Le lion, le coq et la fleur de lys: l'Angleterre et la France dans la culture politique du Québec (1760-1920)», dans Y. Lamonde et Gérard Bouchard, dir., La nation dans tous ses États. Le Québec en comparaison (à paraître). À propos de la conscience coloniale et internationale de l'historien Garneau: Y. Lamonde, "'L'ombre du passé': François-Xavier Garneau et l'éveil des nationalités», dans Gilles Gallichan et Kenneth Landry, dir., [Actes] du colloque Garneau, à paraître en 1997. 
dans aucune autre partie de ses dépendances [...]. Mais voyez le changement. George III, un souverain révéré pour son caractère moral, l'application à ses devoirs royaux et l'amour de ses sujets, succède à Louis $\mathrm{XV}$, un prince méprisé à bon droit pour ses débauches, son indifférence envers les besoins du peuple, ses prodigalités à même le trésor public, au bénéfice de ses favoris et de ses maîtresses. De ce jour, le règne de la loi remplace celui de la violence ${ }^{3}$.

Papineau se félicite encore de l'octroi de la constitution anglaise au Canada en 1791 et reconnaît «notre attachement à cette Constitution qu'un Gouvernement très éclairé nous a donnée dans un âge très éclairé». Au moment du projet d'Union de 1822, il évoque «l'honneur du gouvernement» qui octroya la constitution à la colonie et «le bonheur du peuple qui la recevait», pourfendant les auteurs de ce projet d'Union, ces «Pigmées [qui] ont prétendu attaquer la magnifique fabrique élevée en 1791 par la main des Géants ${ }^{4} »$. Papineau réclame les mêmes droits pour les coloniaux que pour les métropolitains de la Grande-Bretagne, «enfants du même souverain [...] qu'ils demeurassent à Londres [ou] à Québec». S'il insiste pour affirmer que «nous sommes nés sujets anglais aussi bien que ceux qui nous viennent des bords de la Tamise», c'est pour pouvoir réclamer les droits et tous les droits des sujets anglais 5 .

Cette proclamation de fidélité des Canadiens et de Papineau à l'endroit de l'Angleterre avec, à l'occasion, le rappel de l'attitude des Canadiens en 1774 et en 1812, s'impose d'autant plus que, depuis 1810 , la métropole devient de plus en plus le recours obligé d'une colonie où la majorité canadienne-française désespère progressivement de trouver une solution locale aux problèmes coloniaux. Dans le contexte de ces attentes à l'égard de Londres proportionnelles aux déceptions et désillusions qui viennent avec les solutions coloniales annoncées par le Colonial Office et par les gouverneurs, Papineau explique que «l'anglicisation» qui lui est acceptable est celle à la-

3. Louis-Joseph Papineau [LJP], «Élection du Quartier Ouest», $1^{\text {er }}$ juillet 1820 , Le Canadien, 19 juillet 1820; voir aussi «Parlement provincial du Bas-Canada [PPBC]: [Adresse au gouverneur général], 31 janvier 1816», La Gazette de Québec, $1^{\text {er }}$ février 1816. Nous avons mis au point avec Claude Larin la bibliographie des écrits imprimés et des interventions publiées de Papineau et préparons une anthologie de ces textes. Une même intervention de Papineau a pu paraître dans plus d'un journal; nous ne donnons ici qu'une référence.

4. LJP, «Aux Libres Électeurs du Quartier Ouest de Montréal», 24 mars 1820, Le Spectateur canadien, 25 mars 1820; LJP, «Discours prononcé... au dîner patriotique du mois d'octobre», 7 octobre 1822, Le Spectateur canadien, 19 et 26 octobre 1822; voir aussi LJP, «PPBC: [Pétition pour un surcroît de taxes sur les colporteurs et petits merciers], 2 mars 1819», Le Spectateur canadien, 20 mars 1819.

5. LIP, «PPBC: [Droits sur les bois], 11 février 1818», Le Spectateur canadien, 7 mars 1818; LJP, note 4 , deuxième référence. 
quelle s'attend Londres et non pas celle que recherche l'autorité coloniale guidée par les anglophones de la colonie:

Angliciser le pays signifie pour eux priver la grande majorité des habitants de cette province de tout ce qui est cher aux hommes: leurs lois, leurs usages, leurs institutions et leur religion. [...] La Grande-Bretagne ne veut d'autre moyen d'angliciser cette colonie que celui que lui procurent le loyalisme et l'affection de ses habitans; elle ne veut d'autre race britannique que celle qui se compose de sujets britanniques de naissance, loyaux et affectueux ${ }^{6}$.

L'anglicisation politique, celle de l'acquisition et de la promotion des «libertés anglaises», certes, mais l'anglicisation culturelle, non. Admiration et reconnaissance font qu'au lendemain de l'échec du projet d'Union de 1822, le chef du Parti canadien se dit toujours d'accord pour «une autorité suprême au centre de l'empire ${ }^{7}$ ».

\section{ET LA FRANCE?}

De surcroît, cette admiration avouée et répétée de l'Angleterre s'accompagne sinon d'un silence, du moins d'une attitude très réservée à l'égard de la France, l'ex-mère patrie et le symbole de l'identité culturelle des Canadiens français. Papineau participe à cette légende noire qui enveloppe Napoléon au Bas-Canada. Moussée par l'autorité politique britannique métropolitaine et coloniale, entretenue par l'Église catholique romaine, cette représentation fait de «Buonaparte» le «Corse» responsable du saccage de l'Europe et le continuateur d'une certaine Terreur. Papineau se réjouit de la chute de Napoléon et des victoires britanniques ${ }^{8}$ et ce britannisme se double, on l'a vu, d'une représentation de la Nouvelle-France, symbole de "violence» administrative, et d'une Angleterre porteuse du «règne de la loi». Cette négation du caractère français est récurrente. Au moment du projet d'Union de 1822 , alors qu'il précise ce qu'il conçoit être une «anglicisation» acceptable, Papineau se défend, dans une lettre au sous-secrétaire d'État aux colonies, d'un «prétendu attachement à la France et aux principes français ${ }^{9} »$. On pourrait présenter cette prise de

6. LJP à Wilmot, 16 décembre 1822, dans Arthur G. Doughty and Norah Story, Documents relatifs à l' histoire constitutionnelle du Canada (1819-1828) (Ottawa, imprimeur du Roi, 1935), 146-148.

7. «Observations de MM. Papineau et Neilson sur le projet de réunir les législatures du Haut et du Bas-Canada», 10 mai 1823, dans Thomas Chapais, Cours d'histoire du Canada (Québec, Librairie Garneau, 1933), 3: 265.

8. LJP, note 3 , deuxième référence.

9. Voir note $6,147$. 
distance à l'égard de la France comme un procédé rhétorique ou comme une restriction mentale stratégique. Ce déni va plus loin; il recèle d'abord une «canadianisation» de ces anciens colons de France venus en Nouvelle-France et une mémoire d'un «abandon» attribuable à la politique coloniale de la France au XVIII ${ }^{\mathrm{e}}$ siècle. Il dénote aussi une perception de 1789 et surtout de 1793 et de ses dérives. Non pas que Papineau ait de la difficulté à faire siens des acquis de 1789; Papineau et les parlementaires canadiens-français intègrent bien et rapidement les exigences de la démocratie parlementaire et, habitants du Nouveau Monde, ils peuvent refuser 1793 au nom de 1688 et de 1776. Ainsi, Papineau et les hommes publics du Bas-Canada n'ont aucune raison de valoriser leur héritage de Français, mais plutôt leur héritage de langue française et de mœurs face à une population anglocoloniale qui, depuis Craig, utilise cette prétendue «francophilie» pour entretenir un antagonisme politiquement rentable.

Après 1830, donc après les «Trois Glorieuses» journées de juillet, il est révélateur que les 92 Résolutions bas-canadiennes ne contiennent rien sur la France. Papineau évoque même les révolutions nationales comme «le progrès d'un fléau destructeur en diverses contrées de l'Europe» dont il faut «prévenir l'introduction en cette Province ${ }^{10}$ ». Encore en 1835, alors qu'on essaie malicieusement en métropole et dans la colonie de lier les «Conventions» que Papineau propose d'organiser sur le modèle étatsunien à la "Convention» radicale de 1792 en France, celui-ci ridiculise l'accusation «comme s'il y avait lieu de supposer qu'un pareil état de choses pût exister» au Bas-Canada ${ }^{11}$.

\section{L'AMÉRIQUE ET LES ÉTATS-UNIS}

Les États-Unis importent dans la géopolitique de Papineau au sens où ils représentent le verso d'un recto britannique. Cette perception est nouvelle, car jusque vers 1830, les Américains sont vus comme ceux qu'on a, malgré tout, repoussés en 1774 et en 1812, comme des hommes calculateurs et mercantiles et comme de potentiels loyalistes susceptibles de monter dans les «Cantons» et d'en occuper les terres. Papineau connaît les États-Unis pour y avoir voyagé et séjourné et

10. LJP, «PPBC: Adresse en réponse à la harangue d'ouverture de la session, 18 novembre 1831», Le Canadien, 19 novembre 1831; une étude sur les réactions de la presse bascanadienne à la Révolution de juillet 1830 en France manque à notre compréhension de la période.

11. LJP, «PPBC: [Nommer un agent par résolution, M. Roebuck], 17 novembre 1835», La Minerve, 23 novembre 1835. 
d'après les lectures qu'il a pu faire à propos de ce pays grâce à sa bibliothèque personnelle ou à celle de la Chambre d'assemblée ${ }^{12}$. Il reconnaît, par exemple, la qualité des façons de faire étatsuniennes à propos des recensements de la population et, comme des membres de la classe marchande, il se dit constamment favorable aux avantages d'une navigation libre entre le Canada et les États-Unis ${ }^{13}$.

Il serait réducteur et trop simple d'avancer que Papineau joue les États-Unis contre l'Angleterre. La construction de sa représentation de la constitution et de la vie politique étatsuniennes est trop étalée dans le temps et faite à partir d'éléments trop divers pour que son américanité et son républicanisme ne soient que la résultante d'un opportunisme politique. À vrai dire, ce sont les deux questions litigieuses par excellence des luttes constitutionnelles qu'il mène comme député, orateur de la Chambre et chef du Parti patriote qui lui ouvrent les yeux sur l'expérience américaine.

Dès 1822 , avant même que le projet d'Union ne soit connu et au moment où la question des subsides et de la liste civile est devenue le cheval de bataille de la Chambre d'assemblée, Papineau refuse que la liste civile soit votée «pour la vie du Roi» parce que la situation politique de l'Angleterre ne présente «pas d'analogie» avec celle de sa colonie d'Amérique du Nord. La volonté de contrôle de la Chambre d'assemblée sur les dépenses et revenus publics passe par un droit de regard sur la liste civile, qui est aussi un mécanisme de patronage utilisé au détriment des Canadiens français; elle passe aussi par le rappel fait à l'Angleterre que cette question de taxation des colonies et du pouvoir de taxation conditionnel à la représentation avait préci-

12. Roger Le Moine, «Le catalogue de la bibliothèque de Louis-Joseph Papineau (17861871)», dans Y. Lamonde, dir., L'imprimé au Québec. Aspects historiques (18e-20 siècle) (Québec, Institut québécois de recherche sur la culture, 1983), 167-188; id., Le Catalogue de la bibliothèque de Louis-Joseph Papineau (Ottawa, Université d'Ottawa, Centre de recherche en civilisation canadienne-française, «Documents de travail», $\mathrm{n}^{\circ} 21,1982$ ), xxxi-340 p. R. Le Moine explique pourquoi il est difficile sinon impossible de dater l'acquisition des titres de la collection Papineau et en conséquence de savoir quand tel ou tel ouvrage sur les États-Unis, la Jamaique, l'Irlande, l'Amérique latine ou tout autre sujet a pu être acquis et lu par Papineau. Le regard de Papineau sur les États-Unis n'est pas sans rappeler celui d'Alexis de Tocqueville dont La démocratie en Amérique commence à paraître en 1835. Sur la bibliothèque de la Chambre d'assemblée, Gilles Gallichan, Livre et politique et Bas-Canada (1791-1849) (Sillery, Le Septentrion, 1991), $519 \mathrm{p}$.

13. LJP, «PPBC: [Adresse de l'Assemblée à Son Excellence le gouverneur en Chef, Sir John Coape Sherbrooke et à Son Altesse le Prince Régent], 3 mars 1817», L'Aurore, 17 mars 1817; LJP, «Sur l'adresse du Conseil législatif, relativement à la demande des États-Unis pour la navigation libre du St-Laurent», 6 février 1824, Le Canadien, 11 février 1824. 
sément été l'enjeu de la guerre d'Indépendance étatsunienne en $1774^{14}$.

Mais c'est principalement la critique du Conseil législatif et la demande soutenue de son électivité qui, depuis 1827, révèlent l'itinéraire étatsunien et républicain de Papineau. Prenant appui sur l'électivité du Sénat aux États-Unis, Papineau généralise son argumentation et développe l'idée d'un Nouveau Monde différent de l'Ancien, d'un continent américain différent de l'Europe. Dès 1827, il reprend son opposition au vote de la liste civile «pour la vie du Roi» et y ajoute une critique du Conseil législatif colonial dans lequel on prétend, depuis 1791, constituer une aristocratie locale, une Chambre des Lords coloniale. Pour le chef du Parti patriote, ces prétentions n'ont pas de sens en Amérique où l'aristocratie et le Roi «ne se fixent pas au milieu de nous». Une "noblesse» dans le Nouveau Monde constituerait un mimétisme politique et constitutionnel ridicule: «Le Canada est la seule partie de l'Amérique où il existe une espèce de noblesse pour la vie. Ce n'est qu'une singerie, car ces pairs ne peuvent pas être comparés aux pairs anglais plus que le singe peut être comparé à l'homme ${ }^{15}$.» En Amérique, la différence sociale n'est pas créée par la naissance mais par l'éducation, le talent et l'industrie. À la question qu'il pose: «Est-ce dans l'état des mœurs et des usages en Angleterre, ou dans ceux des provinces voisines et des états d'Amérique, que nous devons chercher des exemples pour nous guider?», Papineau répond:

Il faut nécessairement qu'il y ait un roi en Europe où il est entouré de monarchies [...]. Il n'en est pas de même ici, nous n'avons, nous ne pouvons avoir d'aristocratie: nous n'avons pas besoin de ces magnifiques attributs. Nous avons besoin d'un gouvernement simple, tel que celui des États-Unis [...]. De longtemps nous n'aurons pas la force de supporter la splendeur royale et l'aristocratie, l'apanage [d'un] brillant empire canadien [....] $]^{16}$.

Lors d'une intervention parlementaire décisive de 1833, Papineau, qui refère longuement à l'histoire des États-Unis et fait «de grandes louanges [du] système républicain», précise on ne peut plus clairement son projet: «Que demandons-nous de plus maintenant que ce qui a été accordé purement par chartes royales à plusieurs des anciennes colo-

14. LJP, «PPBC: [Adresses de l'Assemblée à la très excellente Majesté du Roi et au gouverneur George Comte de Dalhousie], 25 janvier 1822», La Gazette de Québec, 31 janvier 1822; LJP, «PPBC: Seigneurs et Censitaires, 9 février 1824», La Gazette de Québec, 12 février 1824.

15. LJP, «Discours prononcé... à l'ouverture de l'élection pour le Quartier Ouest de la ville de Montréal», 11 août 1827, La Minerve, $1^{\text {er }}$ octobre 1827.

16. LJP, «PPBC: Composition des Conseils, 16 janvier 1832», La Minerve, 2, 9, 13 février 1832. 
nies? Une constitution républicaine. Nous ne réclamons que ce qui a été accordé aux anciennes colonies américaines ${ }^{17}$.»

Le contenu des 92 Résolutions de février 1834 est éloquent du point de vue de l'américanité de Papineau et des Patriotes. Ces 92 doléances, ébauchées par Papineau, rédigées par Augustin-Norbert Morin et présentées en Chambre par Elzéar Bédard, résument vingt ans de luttes constitutionnelles et constituent le programme électoral du Parti o£Triote pour l'élection de 1834. Londres mettra trois ans et mandatera une Commission présidée par lord Gosford pour y donner réponse avec les Dix résolutions de lord Russell de mars 1837. Les «résolutionnaires» y réitèrent certes la fidélité des Canadiens français à l'Angleterre et à la constitution britannique; mais après une trentaine de résolutions sur l'inacceptabilité du Conseil législatif tel qu'alors constitué, le texte des 92 Résolutions multiplie les allusions «au pays voisin», présenté comme modèle politique contre les abus et comme paradis de l'électivité des institutions publiques. On n'y exclut pas que les colonies britanniques de l'Amérique du Nord fassent un jour ce que firent les Treize colonies en $1776^{18}$. Papineau déclare alors en Chambre: «Il est certain qu'avant un temps bien éloigné, que toute l'Amérique doit être républicaine. [...] Il ne s'agit que de savoir que nous vivons en Amérique, et de savoir comment on a vécu ${ }^{19}$.»

Papineau n'est pas américain qu'en paroles; il l'est aussi en actes, tout comme les Patriotes: on organise des comités de correspondance sur le modèle des révolutionnaires étatsuniens, Papineau propose la tenue de «conventions» pour formuler une nouvelle constitution, les Fils de la liberté seront bientôt mis sur pied à la manière des Sons of Liberty et, surtout, Papineau radicalise sa position sur l'électivité du Conseil législatif en juillet 1834, en mettant de l'avant une stratégie de non-consommation de biens importés d'Angleterre que les insurgés étatsuniens avaient fait leur en 1774. Tout en privant le gouvernement colonial de revenus, le Bas-Canada montrait une volonté d'autonomisation en explorant les possibilités d'une autosuffisance plus

17. LJP, «PPBC: Sur les diverses références relatives à la composition du Conseil législatif, 10 janvier 1833», Le Canadien, 16, 18, 23 janvier 1833.

18. Texte des 92 Résolutions du 17 février 1834, dans Narcisse-Eutrope Dionne, Les trois comédies du "statu quo» 1834 (Québec typ. Laflamme et Proulx, 1909), 127-235; voir les résolutions $31,41,43,45,46,48,50,56$.

19. LJP, «PPBC: État de la province. lère résolution, 18 février 1834», La Minerve, 27 février et 3 mars 1834 . 
grande grâce à l'exploitation de «la toison des bêtes» pour les vêtements, de brasseries et de distilleries pour l'alcool ${ }^{20}$.

Et alors que, selon Papineau, des centaines d'individus qui voyagent aux États-Unis constatent l'avance de l'Amérique indépendante sur l'Amérique coloniale, Downing Street n'a pas compris et ne veut pas comprendre l'état social de l'Amérique ${ }^{21}$.

Cette référence marquée aux États-Unis ne fait pas pour autant de Papineau un partisan de l'intégration aux États-Unis. Le chef du Parti patriote parle encore au conditionnel; à l'annonce des résolutions de lord Russell, Papineau affirme: «Cette union [américaine] est séduisante, et la nôtre dans le moment actuel est humiliante. Est-ce à dire que de suite nous devons répudier l'une, pour épouser l'autre? Doucement! Si cet arrangement était le seul qui pût rétablir la paix du ménage, oui, il faudrait y avoir recours.» Du même souffle, il lance un nouvel avertissement au pouvoir britannique: «S'il est bien clair et bien établi, que la détermination de lord Russell est un plan fixe et arrêté auquel il donnera suite dans l'avenir [...] que les colonies sont conservées, non dans l'intérêt réciproque des peuples, mais dans celui du patronage et de la corruption ministérielle, l'histoire des anciennes plantations recommencera avec le même résultat inévitable.» Le même résultat? En mai 1837, Papineau a toujours intérêt à raviver la mémoire de 1776 et l'histoire des États-Unis. Il a toutefois une vision particulière du mode d'union des États au sud du $45^{\mathrm{e}}$ parallèle, vision qui est aussi un scénario de ce que serait l'expérience politique différente d'un Bas-Canada intégré aux États-Unis:

Les États-Unis ne peuvent avoir de colonies. Leur constitution pourvoit d'avance à ce qu'un territoire, dès qu'il a 60000 habitants, puisse se constituer en état libre et indépendant. Il devient le maître et l'arbitre absolu de son sort. Il n'a pas à craindre la nomination d'Officiers, qui y seraient envoyés passagèrement, pour s'enrichir au galop, et aller digérer d'énormes richesses mal acquises à mille lieues de distance; pour solliciter du gouverneur

20. [LJP], Observations sur la réponse de Mathieu lord Aylmer à la députation du Tattersall..., le 15 avril 1834... (Montréal, imprimées sous la direction du Comité central et permanent du district de Montréal, juillet 1834), 25-32 ou Institut canadien de microreproductions historiques [ICMH], no 39135; LJP, «Discours de l'Honorable Louis-Joseph Papineau à l'assemblée du comté de Montréal tenue à Saint-Laurent, le 15 mai [1837]..., $\mathrm{La}$ Minerve, 25 et 29 mai 1837 ou ICMH, $\mathrm{n}^{\circ}$ 21619; LJP, «[Assemblée de Sainte-Scholastique,

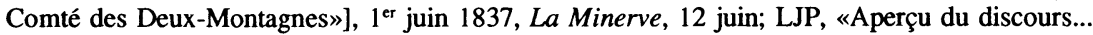
à l'assemblée des comtés de L'Assomption et de Lachenaie», 29 juillet 1837, La Minerve, 10 août 1837.

21. LJP, «PPBC: Contingens, 7 mars 1835», La Minerve, 19 mars 1835; LJP, «Traduction de la lettre de M. Papineau accompagnant les résolutions de la Chambre d'Assemblée...», 16 mars 1836, La Minerve, 5 mai 1836. 
général, qu'il intervienne et donne à des monopoleurs étrangers les terres de l'état à un tiers du prix auquel il les vendra aux citoyens résidens; pour qu'il dépouille la législature locale du droit de régler toutes ses dépenses locales quand et comme elle l'entendra; pour qu'il altère et refasse les lois et coutumes locales sans y rien comprendre, et porte l'insécurité dans la jouissance des propriétés et l'incertitude dans l'administration de la justice.

Pour Papineau, les vingt-six États alors constitués aux États-Unis forment «vingt-six souverainetés indépendantes» qui connaissent une prospérité qu'elles n'auraient pas connue «si elles eussent demeurées [sic] dans la dépendance et la servitude coloniale ${ }^{22} \gg$. Serait-ce là l'avancée ultime de la «souveraineté» que Papineau pouvait avoir à l'esprit?

L'analyste ne peut évoquer les propos de Papineau sur la «différence sociale» en Amérique, sur «l'orientation sociale» en Europe, sur le choix de la simplicité américaine contre la splendeur européenne ou sur l'absence «d'aveugle déférence» pour les titres et la naissance en Amérique sans scruter les rapports entre le refus d'une aristocratie anglaise locale, d'une part, et le statut de seigneur de Papineau, d'autre part. Il faut toutefois rappeler que, pour Papineau, la seule aristocratie naturelle est celle qui est due à l'éducation, au talent et à «l'industrie ${ }^{23}$ ». Tout seigneur qu'il soit de 1817 à l'abolition du régime seigneurial en 1854 - avec les absences annuelles de la seigneurie du député et de l'orateur de la Chambre et l'exil de 1837 à 1845 - , misant sur les revenus de la rente des censitaires et de la rente des entrepreneurs forestiers, capitalisant sur l'endettement paysan pour construire le manoir de Montebello vers 1850, Papineau devait, à ses propres yeux et à ceux des électeurs et des Patriotes, concilier ce statut de seigneur et ses positions de démocrate et de républicain. Pour pouvoir croire à la propriété, à la liberté, à l'égalité et à la fraternité, Papineau devait adhérer à l'idée d'une certaine mobilité sociale. Il reconnaît «le besoin du siècle» d'atteindre «au bonheur social», la nécessité du rapprochement des classes et de la destruction des «aristocraties superficielles». Refusant en 1819 de voter un projet de loi qui taxerait les merciers et les colporteurs, il se dit admiratif de l'excellent principe de la constitution anglaise qui permet «aux petits de s'élever». En 1833, il déclare en Chambre: «La masse du genre humain commence à s'apercevoir qu'elle a droit de s'élever au rang des hommes, et que la noblesse hériditaire née que

22. LJP, note 20 , deuxième référence ou ICMH, $\mathrm{n}^{\circ} 21619,10-11$.

23. LJP, note 16; Claude Baribeau, La seigneurie de la Petite-Nation (1801-1854). Le rôle économique et social du seigneur (Hull, Éditions Asticou, 1983), 166 p. 
pour consommer les fruits de l'industrie des autres doit bientôt tomber.» À la veille des rébellions, il affirme que, dans les circonstances, «le seigneur n'est pas plus que son censitaire ${ }^{24} »$.

Il n'est pas évident que le paradoxe de la position sociale de Papineau puisse être levé; démocrate et inconditionnel de la souveraineté populaire, le républicain demeure toutefois confronté à la dimension sociale du républicanisme, à son exigence d'égalité de droit sinon de fait. Si le républicanisme liquide la monarchie et l'aristocratie, Papineau ne peut pas ne pas se savoir seigneur. Mais sa position républicaine ne peut être réduite à un antibritannisme primaire. Il faut reconnaître un itinéraire républicain chez Papineau: l'homme qui, en 1820 , au décès de George III, compare avantageusement celui-ci à Louis XV et l'homme qui déclare, au moment des 92 Résolutions, que toute l'Amérique doit être républicaine, a manifestement évolué.

On ne peut toutefois pas invoquer ici le fait qu'à titre de chef du Parti patriote, il n'ait pas demandé le suffrage universel, dans une société européenne et américaine où la chose était peu courante; mais on doit aussi rappeler qu'il a déclaré, dans une société où le suffrage est passablement général: "La démocratie est l'essence de la société ici.» C'est la souveraineté populaire, l'exercice libre du droit de vote, qu'il défend lorsqu'il s'indigne des événements du 21 mai 1832 et de l'attitude du gouverneur. S'il vote en faveur d'une indemnité pour les députés, c'est qu'elle permettra à tout citoyen à revenu modeste d'être éligible et de siéger à Québec ${ }^{25}$. Son argumentation soutenue de la prépondérance de l'Assemblée élue sur le Conseil nommé et son combat constant, à la suite de celui du Parti canadien, pour la séparation des pouvoirs législatif et judiciaire sont autant de signes de son sens démocratique, tout comme sa défense de la tolérance religieuse et sa volonté, contrée par le Conseil législatif, d'introduire dans l'administration des biens paroissiaux de l'Église le principe de la taxation et de la représentation pour les notables. C'est toujours ce sens des moyens démocratiques qui fait opter Papineau et le Parti patriote pour les assemblées populaires et pour l'idée étatsunienne des conventions.

\section{L'EUROPE ET L'ANCIEN MONDE}

La perception de l'Europe par Papineau est à l'avenant, congruente avec sa promotion de l'Amérique et sa critique de l'Angle-

24. LJP, «Allocution lors du dîner offert à l'Honorable D. B. Viger», 9 mai 1831, Le Canadien, 14 mai 1831; LJP, note 4, dernière référence; LJP, note 17; «La voix du peuple [assemblée de Berthier], 18 juin 1837, La Minerve, 22 juin 1837.

25. LJP, «PPBC: Conclusion des débats de lundi dernier, 15 février 1836», La Minerve, 29 février 1836; «PPBC: Affaire du 21 mai, 11 décembre 1832», La Minerve, 17 décembre 1832; «PPBC: Bill d'indemnité, 4 novembre 1835», La Minerve, 12 novembre 1835. 
terre et de la France. Les institutions qui conviennent au Bas-Canada doivent être autres que celles de l'Europe car «[c]e n'est donc pas de la mère patrie non plus que dans le reste de l'Europe, où l'orientation sociale est tout à fait différente, où la répartition des richesses est fort inégale, qu'on doit chercher des exemples; c'est plutôt en Amérique où l'on ne voit ni fortune colossale, ni pauvreté dégradante, où l'homme de génie parcourt les différents rangs sociaux sans obsta$\mathrm{cle}^{26}$ ». Pour Papineau, «toutes les opinions, tous les préjugés de la vieille Europe viennent tomber auprès du républicanisme de l'Union»; il se perçoit alors à ce point américain qu'il peut déclarer: «il n'y avait rien ici de cette aveugle déférence qu'on a en Europe pour les titres et la naissance, qui font tant ressortir l'arrogance et l'orgueil de ceux qui viennent parmi nous de l'ancien continent, et qui pensent que l'humiliation à laquelle ils s'y sont soumis doit être compensée ici, pour eux, en exigeant la même servilité à leur égard ${ }^{27}$.» Les «récemment déballés d'Europe» ne l'impressionnent pas nécessairement et il écrit à propos des membres de la commission Gosford, dont on a appris qu'elle avait déjà ses directives et ses conclusions avant de quitter Londres: «Il n'est pas nécessaire qu'on nous envoie de temps en temps quelque sage d'Europe pour nous éclairer dans la démarche qu'il nous convient d'adopter ${ }^{28}$.»

\section{LA RÉFÉRENCE AU ROYAUME-UNI ET AUX AUTRES COLONIES ANGLAISES}

Lorsqu'il déclare: «C'est donc dans l'histoire des autres colonies anglaises qu'il faut chercher ce qui nous convient ${ }^{29}$ ", Papineau est conséquent avec lui-même. C'est ce qu'il a fait depuis la décennie 1820 par ses références constantes aux États-Unis, ex-colonies anglaises qui ont fait leur indépendance. C'est ce qu'il continue de faire en s'inspirant de l'histoire de l'Irlande, de la Jamaïque, de l'Écosse, de Malte, du Haut-Canada et des autres colonies anglaises d'Amérique du Nord (Nouvelle-Écosse, Nouveau-Brunswick). Le chef du Parti patriote a souvent le réflexe de comparer les colonies américaines aux autres colonies de l'Empire, signalant, par exemple, que la liste civile bas-canadienne n'a pas d'équivalent dans l'Empire britannique, ou se

26. LJP, note 17 et LJP, note 25 , dernière référence.

27. LJP, notes 19 et 16 .

28. LJP, «État du pays», 8 février 1831, La Minerve, 12 mars 1831; LJP, «PPBC: Accusation de M. le Juge Gale, 18 février 1836», La Minerve, 3 mars 1836. Sur l'événement et ses significations, France Galarneau, «L'élection partielle du quartier-ouest de Montréal en 1832: analyse politico-sociale», Revue d' histoire de l'Amérique française, 32,4 (mars 1979): 565-584.

29. LJP, note 19. 
préoccupant des effets négatifs pour les autres colonies anglaises de toute concession faite par le Bas-Canada ${ }^{30}$.

La référence à l'Irlande est sans doute la plus fréquente chez Papineau entre 1827 et 1837 . Il faut rappeler que la trame irlandaise de l'évolution du Bas-Canada est tout à fait contemporaine des grandes revendications de O'Connell et de sa lutte pour l'émancipation des catholiques (1829); elle correspond aussi à une phase de démarrage de l'émigration irlandaise vers la colonie canadienne et à une prise en compte de plus en plus obligée de l'électorat irlandais par le Parti patriote et par le Parti bureaucrate. C'est au moment de l'élection de 1827, alors que le Canadian Spectator (1822) de Jocelyn Waller paraît déjà et que La Minerve (1826) vient d'être lancée, que le groupe irlandais prend de l'importance. Daniel Tracey organise les Friends of Ireland et lance le Irish Vindicator (1828) bientôt financé par des Canadiens français. L'enjeu irlandais est manifeste lors de l'élection partielle et meurtrière dans la circonscription de Montréal-Ouest du 21 mai 1832, alors que le docteur Edmund O'Callaghan prend la relève de Tracey et devient le bras droit de Papineau ${ }^{31}$.

On ne met guère de temps à établir la comparaison entre Papineau et $\mathrm{O}^{\prime}$ Connell dans la presse étatsunienne ou à la Chambre des communes de Londres, où le député et ami de Papineau, Joseph Hume, compare l'influence de celui-ci au Bas-Canada à celle de O'Connell en Irlande ${ }^{32}$. Papineau lui-même se dit admiratif d'hommes politiques comme John Arthur Roebuck et O'Connell «qui vouent leur existence au salut du genre humain» et sur lesquels comptent les Bas-Canadiens:

[Que ceux-ci] avaient la garantie de cet ami de la race humaine, O'Connell, qu'ils auraient une réforme et que les promesses d'un tel homme devaient être d'un poids incontestable. O'Connell [...] promit l'émancipation à 7000000 de ses compatriotes et ne la leur gagna-t-il pas? [...]. Il garantit l'abolition des titres, et ils sont

30. LJP, «PPBC: État de la province, 19 août 1837», La Minerve, 11 septembre 1837; LJP, «Traduction de la lettre de M. Papineau, accompagnant les résolutions de la Chambre...», 16 mars 1837, La Minerve, 5 mai 1837.

31. Mary Finnegan, «Irish-French Relations in Lower-Canada», Sessions d'études. Société canadienne d'histoire de l'Église catholique, 52 (1985): 35-49; Robert Charles Daley, Edmund Bailey O'Callaghan: Irish Patriot, Ph.D. (History), Concordia University, 1986, xi483 p.; Jack Verney, O'Callagnan: the Making and Unmaking of a Rebel (Ottawa, Carleton University Press, 1994), 178 p.; Maurice Lemire, «Les Irlandais et la rébellion de 1837-8», British Journal of Canadian Studies, 10,1 (1995): 1-9; Maureen Slattery, «Irish Radicalism and the Roman Catholic Church in Quebec and Ireland (1833-1834): O'Callaghan and O'Connell Compared», Canadian Catholic Historical Association, Historical Studies, 63 (1997): 29-58.

32. LJP, «Troubles en Canada et assemblée de Saint-Benoît», 19 juin 1837, La Minerve, 6 juillet 1837; British Parliamentary Debates, Third Series, 16 mai 1836, 33: 942. 
à la veille d'être abolis. Il leur donna sa parole que l'union serait révoquée et il réussira [...]

Pour le chef du Parti patriote qui se bat depuis 1809 et qui a aussi vu se succéder les ministres, «les promesses d'un tel homme, qui a délivré sa patrie des fers qui lui étaient imposés, et qui a balayé devant lui ministères après ministères, sont une assurance que le Canada aussi recevra bientôt la réforme qu'il avait demandée dans ses institutions et son gouvernement ${ }^{33}{ }^{\prime}$. O'Connell, qui appuie à la Chambre des communes les positions du Bas-Canada, sert à Papineau d'écran de projection des revendications des Patriotes:

Que puisque des jours de paix et de justice sont promis à l'Irlande, dont le pillage et l'oppression pendant des siècles, ont gorgé et déshonoré l'Aristocratie Britannique, à laquelle le libérateur de son pays et l'ami du nôtre, O'Connell, arrache enfin cette proie si riche, cette victime si grasse, ils seront donnés aussi aux Canadiens. O'Connell a déjà renversé Stanley, l'oppresseur, il renverserait de même son successeur, si lui aussi voulait un gouvernement qui eut pleine puissance et autorité sur le peuple, et non un gouvernement qui tire son autorité du peuple. C'est un tel gouvernement que la colonie doit avoir: désormais elle n'en doit ni ne peut en supporter d'autre ${ }^{34}$.

Au-delà de la personne et du symbole de O'Connell, la situation irlandaise permet à Papineau des comparaisons qui deviennent raisons. À l'élection de 1827, il met en valeur l'esprit d'indépendance des Irlandais et le fait que leurs concitoyens des colonies partagent les mêmes intérêts que les Bas-Canadiens. Faisant allusion au décès de trois Canadiens lors de l'émeute électorale du 21 mai 1832, le chef du Parti patriote déclare: «Il est clair que pour les juges Bretons du District de Montréal le sang d'un Canadien est aujourd'hui de la même valeur que l'était celui d'un Irlandais Catholique, devant un juge et un jury orangistes, avant l'émancipation catholique.» L'exemple irlandais permet aussi à Papineau de préciser les demandes politiques et constitutionnelles du Bas-Canada; il affirme après le vote des 92 Résolutions, dans un très rare passage où il utilise l'épithète «national»: «Un gouvernement local responsable et national pour chaque partie de l'Empire, quant au règlement de ses intérêts locaux, avec une autorité de surveillance dans le gouvernement Impérial, pour décider de la paix et de la guerre et des relations de commerce avec l'étranger: c'est là

33. LJP, «Discours prononcé... aux hustings du Quartier-Ouest», octobre 1834, La Minerve, 30 octobre 1834 .

34. LJP, «À Messieurs les Électeurs du Quartier Ouest de Montréal», 20 octobre 1834, La Minerve, 20 octobre 1834. 
ce que demandent l'Irlande et l'Amérique britannique ${ }^{35}$.» La signification du "gouvernement local responsable» parait claire; celle d'un «gouvernement national pour chaque partie de l'Empire» ne précise guère si la «partie de l'Empire» réfère au tout, Bas-Canada et HautCanada, ou à la partie, le Bas-Canada.

Lorsque le ton a monté après la nouvelle des résolutions Russell de mars 1837, l'orateur de la Chambre évoque l'histoire des ministres des Colonies et «l'inconséquente contradiction qu'il y a dans leur politique, qui à la fin et après des siècles d'oppression contre l'Irlande infortunée, devient libérale, parce que l'Irlande se fait craindre; qui est si basse et si rampante vis-à-vis de la Russie, qui aussi se fait craindre; et qui est si injuste, arrogante, et dédaigneuse à l'égard du Canada, qu'ils ne craignent point ${ }^{36}$ ». Il avait déjà dénoncé le colonialisme anglais à l'égard de l'Irlande: «Une nation n'en sut jamais gouverner une autre. Les affections Bretonnes pour l'Irlande et les colonies, n'ont jamais été que l'amour du pillage de l'Irlande et des colonies, abandonnées à l'exploitation de l'aristocratie bretonne et de ses créatures.» L'homme qui avait connu tous les secrétaires d'État aux Colonies depuis le gouverneur Craig savait ce que certains d'entre eux pouvaient réserver à l'une d'elles: «Car il n'y a pas de doute que l'homme pétulant et hautain qui est à la tête du bureau colonial, M. Stanley, qui a asservi l'Irlande, sa patrie, par une loi barbare et inhumaine, voudrait nous donner quelque chose de semblable.» Il craignait que «le Tyran de l'Irlande» ne devienne «l'oppresseur du Canada». A propos de l'expérience irlandaise du duc de Richmond, il déclare: "Quand je pense qu'un duc de Richmond, qui avait commandé l'Irlande en qualité de vice-roi, où un sentiment d'orgueil national l'environnait tous les jours, au milieu de Dublin, de la pompe et de l'éclat de la Royauté, et que cet homme, après avoir abandonné ce théâtre brillant, s'en vint ici pour réparer les débris de sa fortune $[\ldots]^{37} \gg$.

Les ressemblances soulignées entre l'Irlande et le Bas-Canada n'empêchent pas les distinctions; par exemple, Papineau insiste pour préciser que l'émancipation des catholiques est une question strictement irlandaise et n'a rien à voir avec les autres colonies, dont le BasCanada, qui a acquis cette émancipation en $1774^{38}$. C'est d'ailleurs cette même question religieuse qui invite Papineau à référer à l'expé-

35. LJP, «Aux Libres et Indépendants Électeurs du Quartier Ouest de Montréal», 3 décembre 1834, La Minerve, 4 et 8 décembre 1834.

36. LJP, note 22 ou ICMH, $\mathrm{n}^{\circ} 21619,13$.

37. LJP, note 35 ; LJP, note 21 , première référence; LJP, note 20 ; note 16 .

38. LJP, note 16 . 
rience de l'Écosse et de Malte. Dans le premier cas, il défend une résolution pour que l'Église d'Écosse et d'autres Églises protestantes dissidentes aient un même accès au produit des réserves du clergé et c'est alors qu'il fait allusion à la loi d'Union entre l'Angleterre et l'Écosse. Dans le cas de Malte, il rappelle que les clauses de la capitulation de cette île avaient fait de la religion catholique la religion établie et de la religion protestante une religion tolérée, preuve qu'une métropole anglaise et protestante savait et devait faire les différences qui s'imposaient et pouvait préconiser la tolérance religieuse ${ }^{39}$.

Après les États-Unis et l'Irlande, la référence la plus fréquente à l'expérience d'autres colonies anglaises est celle de la Jamaïque. Ce sont les grandes questions constitutionnelles litigieuses qui servent de prétexte à des références à l'histoire de cette colonie. D'abord l'octroi historique d'une Chambre d'assemblée dans les deux colonies: Papineau rappelle que lorsque la Jamaïque fut «arrachée» à l'Espagne, aussitôt on y fit élire des représentants - ce que Murray ne fit pas en 1764 - et que, depuis, la colonie a été gouvernée sans charte royale ou loi du Parlement. L'homme politique, qui connaît bien l'histoire de son pays, explore cette comparaison pour avancer que, devenant sujets du roi d'Angleterre en 1763, les Canadiens avaient immédiatement droit d'élire et d'être élus; mais le «fiel qu'on nourrissait alors» contre les Canadiens et «le machiavélisme qui pour dominer divise» avaient différé l'octroi de ce droit fondamental ${ }^{40}$. S'il lit aussi les journaux jamaïcains pour blâmer le Conseil législatif local, Papineau utilise le cas de cette île antillaise d'abord comme argument contre la liste civile qu'on prétend maintenir au Bas-Canada:

Dans quelle autre colonie a-t-on formé d'aussi singulières prétentions? Dans les anciennes colonies anglaises, dans les îles à sucre et les autres dépendances britanniques, les revenus sont prélevés en vertu d'actes de courte durée. On a vu dans la Jamaïque l'assemblée reconnaître le mérite d'un gouverneur, et y être attaché [sic] au point de statuer que tels actes pour le prélèvement des deniers publics seraient en force aussi longtems que le gouverneur demeurerait parmi eux, mais pas une seule colonie n'a accordé l'octroi pour la vie du Roi.

Le chef du Parti patriote et orateur de la Chambre n'exclut pas qu'on vote les subsides pour une période de temps limitée comme le

39. LJP, «L'adresse suivante, proposée par M. Papineau...», 4 mars 1824, Le Canadien, 17 mars 1824; LJP, note 16.

40. LJP, note 17; LJP, note 30 , première référence. Papineau possédait deux éditions (1793 et 1818-1819) de l'ouvrage de Bryan Edwards, The History, Civil and Commercial of the British Colonies in the West Indies, R. Le Moine, Le catalogue..., op. cit., 251-252. 
fait la Jamaïque, de six mois en six mois, parce que les troupes doivent y être payées, sans quoi la métropole retirerait l'armée et la minorité blanche y serait en danger ${ }^{41}$.

On s'attend enfin à ce que Papineau connaisse la situation politique du Haut-Canada et des autres colonies anglaises d'Amérique du Nord. Il y réfère fréquemment à propos du nombre de députés élus en regard de la population, du combat pour le contrôle des dépenses par la Chambre d'assemblée, de la composition inéquitable du Conseil législatif, du vote annuel des subsides et de la liste civile ou de la langue utilisée dans les cours de justice. L'occasion est parfois d'autant plus éloquente qu'un gouverneur nommé au Bas-Canada a pu se comporter différemment en Nouvelle-Écosse à propos du vote annuel des subsides. L'intention est toujours la même: faire voir la différence dans le traitement métropolitain des différentes colonies ${ }^{42}$.

\section{LES RÉFÉRENCES ABSENTES}

Entre la fin des guerres napoléoniennes et le Traité de Vienne (1815), d'une part, et l'éveil des nationalités après 1830, d'autre part, les colonies américaines de l'Espagne et du Portugal occupent le devant de la scène internationale, aidées dans leurs initiatives par les interventions de Napoléon en Espagne et au Portugal. Bolivar, Miranda, San Martin, O'Higgins, Iturbide deviennent les libérateurs du Venezuela, de l'Argentine, du Chili, du Pérou et du Mexique dans une dynamique d'émancipation coloniale où les tentatives de reconquête des métropoles et les conflits internes aux colonies font durer le processus de rupture. Les nouveaux États d'Amérique latine sont rapidement reconnus par les États-Unis (1822) et par l'Angleterre (1825) qui y ont des intérêts économiques et politiques. Et la «doctrine» (1823) de James Monroe, qui exclut dorénavant toute intervention européenne sur le continent américain, peut aussi permettre de comprendre le regard que Papineau pose de plus en plus sur l'Amérique à la fin de la décennie 1820. Si tel est le cas, on s'explique mal toutefois le peu de références et le type d'allusions qu'il fait à ces nouveaux États d'Amérique latine. Ces références, tardives, vont encore dans le sens de son britannisme et dans le type d'usage qu'il fait des autres expériences coloniales. Papineau explique en 1832 comment la libéralité

41. LJP, «PPBC: Subsides, 19 mars 1831», La Minerve, 24 mars 1831; LJP, «PPBC: Continuation des débats du 22 février. État de la province et subsides, 22 février 1836», Le Canadien, 16 mars 1836.

42. LJP, note 15; "Aux Électeurs du Quartier Ouest de Montréal», 20 août 1827, La Minerve, 23 août 1827; notes 16 et 33; «PPBC: Liste civile, 21 janvier 1832», Le Canadien, 22 février 1832. 
de l'Angleterre a préparé les États-Unis à profiter de l'indépendance, contrairement à ce que les colonies espagnoles ou portugaises ont pu connaître de leurs métropoles. Deux ans plus tard, il reprend cette idée: «Les colonies Espagnoles et Françaises, avec des institutions moins libérales [que celles des ex-colonies devenues les États-Unis], ont été plus malheureuses et ont dû lutter beaucoup contre le vice de ces institutions.» L'exemple mexicain lui permet au même moment de préciser le vice de ces institutions: sous «l'odieuse domination de l'Espagne», les emplois étaient donnés au Mexique à des Espagnols, les vice-rois et les juges choisis parmi des non-coloniaux «étrangers et hostiles à la religion, à la langue, aux lois, aux établissemens, aux mœurs, aux habitudes, aux attachements des créoles». Et l'homme politique qui se bat pour la récupération des biens des jésuites dont les autorités britanniques se sont emparées, ajoute: «et les pouvoirs [britanniques] qui voyaient avec faveur les établissemens consacrés à l'enseignement public dans le Montréal espagnol [Mexico], ne méditèrent jamais la spoliation des établissemens consacrés à l'enseignement public, dans le Montréal Américain ${ }^{43}$.» On ne peut trouver d'exemple plus transparent du type de comparaison entre les colonies habituellement fait par Papineau.

À nouveau, ce britannisme doit s'expliquer en regard de l'évolution de la France et de la perception que Papineau en a. L'histoire politique française contemporaine n'a pas alors l'attrait qu'elle pourra avoir avec les «Trois Glorieuses» journées de juillet 1830. Napoléon connaît son Waterloo, l'exil, la double abdication, et le retour de la monarchie (Louis XVIII puis Charles X) ne présente rien d'attrayant pour un admirateur des institutions britanniques tel que Papineau. Surtout que l'Angleterre, exclue de la «Sainte-Alliance» (Autriche, Prusse, Russie) qui sort du congrès de Vienne mais incluse dans la Quadruple-Alliance des pays qui ont triomphé de Napoléon, est la puissance montante, puissance qui trouvera tout son éclat au moment de la montée sur le trône de la reine Victoria en 1837. Alors que les autres monarchies européennes sont systématiquement remises en cause, l'Angleterre s'affirme et s'impose. Et le Bas-Canada est la colonie de cette métropole impériale.

On peut comprendre qu'à un moment où Papineau est toujours admiratif de l'Angleterre et de sa constitution, il n'ait pas fait référence aux soulèvements de Naples (1820) ou du Piémont italien et à l'expérience de la Grèce qui fait son indépendance (1822) après des siècles de sujétion à la Turquie; la Grèce demande même le protectorat

43. LJP, notes 16,26 et [LJP], note 20 , première référence. 
de l'Angleterre en 1825. Mais l'absence de référence de Papineau, après 1830, aux pays européens qui tentent de s'émanciper de tutelles impériales, demande explication et celle-ci viendra en conclusion. Aucune référence publique à l'expérience de la Belgique qui fait son indépendance en octobre 1830, aucune allusion au soulèvement des libéraux catholiques polonais ni à l'insurrection de Varsovie (18301831), aucune mention des soulèvements de Modène, de Parme et des Romagnes dans ce qui deviendra l'Italie réunie ou de la création par Mazzini de la Jeune-Italie après celle de la Jeune-France. L'homme qui connaissait aussi Lamennais, celui dont on enseignait la philosophie au Collège de Saint-Hyacinthe jusqu'en 1834 et dont on lisait L'Avenir (1830-1831), ne réfère pourtant pas aux Paroles d'un croyant (1834), plaidoyer évangélique de l'émancipation, ou aux $A f$ faires de Rome (1836), dénonciation de la collusion entre Rome, les évêques polonais et la Russie impériale. Il s'agit pourtant de ce même Lamennais dont Papineau sera l'un des proches durant son exil parisien (1839-1845). Mais peut-être ne fallait-il pas jeter de l'huile libérale sur le feu religieux en référant à un émancipateur que Rome condamna pour sa philosophie en 1832 et pour ses idées politiques en 1834.

\section{LA MONTÉE DE L'IMPATIENCE ET LA DÉSILLUSION}

Le ton monte chez Papineau après 1830 en raison du caractère lancinant de la question de la liste civile et de celle du Conseil législatif. Mais le déclencheur de l'éveil à la situation proprement coloniale du Bas-Canada se trouve déjà dans le projet d'Union de 1822 et dans la prise de conscience, lors du voyage à Londres en 1822-1823, d'un destin dorénavant menacé. Le projet d'Acte d'Union est alors perçu comme un «acte d'agression» et les unionistes, explique Papineau, «ont fortifié parmi nous un esprit d'union que rien ne pourra désormais affaiblir ${ }^{44} »$. Si le Comité des Communes sur les affaires du Canada entretient toujours en 1828 des espoirs d'écoute et de suivi de la part de la métropole, l'inaction des secrétaires d'État aux Colonies, qui se succèdent au rythme de six en dix ans, de 1828 à 1837 , et l'attitude des gouverneurs coloniaux et de leurs Conseils exécutif et législatif émoussent les attentes de Papineau.

Déjà en 1827, l'orateur observe que les dissolutions de la Chambre d'assemblée par les gouverneurs sont plus fréquentes au Bas-Canada que dans les autres colonies britanniques d'Amérique du Nord ou

44. LJP, «Discours prononcé... au dîner patriotique du mois d'octobre», 7 octobre 1822 , Le Spectateur canadien, 16 et 26 octobre 1822. 
d'ailleurs ${ }^{45}$. Il est alors clair pour Papineau que certains abus n'ont pas leur pareil dans les autres colonies et que le temps des promesses a trop duré: «Il est tems que les déclarations de la mère patrie si souvent réitérées et accompagnées des mots sonores du 'peuple loyal' dont elle dit vouloir le bonheur, se réalisent.» Le chef du Parti patriote commence à identifier et à nommer la stratégie colonisatrice de l'Angleterre au moment de l'émeute électorale de mai 1832; dans ces événements, selon lui, «il y avait les plus forts indices que l'on considérait le despotisme militaire comme la loi du pays» et qu'il fallait «empêcher le pays d'être battu sous la force et par la crainte ${ }^{46}$ ». À deux occasions, Papineau dégage les intentions qui, selon lui, ont présidé à la mise en place de l'Exécutif et du Conseil législatif qu'expérimente le Bas-Canada depuis 1791: «Aussi n'ont-ils été constitués, je pense, que d'après la maxime des tyrans: 'divisez et régnez' ${ }^{47}$.» En ces années d'éveil des nationalités et de poésie romantique, la référence aux «tyrans» n'est pas banale et Papineau l'utilise à nouveau en affirmant: «On a adopté un système colonial qui n'avait exclusivement en vue que l'avantage des marchands et des capitalistes d'Angleterre» et le «gouvernement ici n'était qu'une tyrannie déguisée ${ }^{48}$ ».

Et puis Papineau commence à se montrer plus critique à l'égard de cette constitution anglaise dont il est si admiratif depuis 1815 et qui octroie aux sujets britanniques des droits qu'il réclame en totalité pour les Bas-Canadiens. À la question: cette «magnifique» constitution anglaise convient-elle au Canada?, il répond qu'elle convient peut-être à un gouvernement indépendant mais pas à une colonie dépendante ${ }^{49}$. S'esquisse alors une revendication coloniale qui parait à la fois radicale et peut-être un peu naïve d'un point de vue constitutionnel: «Que l'Angleterre autorise le pays à réformer lui-même sa constitution, telle serait la seule détermination équitable et prudente qu'elle pourrait adopter ${ }^{50} . »$ Il pouvait être facile, pour le Colonial Office et le Parlement anglais, de rétorquer que seul le Parlement impérial qui avait octroyé la constitution de 1791 pouvait l'amender.

45. LJP, note 15.

46. LJP, «PPBC: Lieutenant Colonel Eden, 28 février 1833», La Minerve, 18 mars 1833.

47. LJP, «PPBC: État du pays [abolition du Conseil législatif], 11 mars 1831 » La Minerve, 24 mars 1831 et note 32 . 1833.

48. LJP, «PPBC: Bill des subsides et la liste civile, [sans date]», Le Canadien, 8 avril

49. LJP, «PPBC: Douzième résolution, 9 mars 1831», La Minerve, 17 mars 1831; LJP, «PPBC: Adresse de l'orateur de la Chambre à Son Excellence, lord Aylmer, 20 novembre 1832», Le Canadien, 21 novembre 1832; note 17.

50. LJP, note 44, première référence; LJP, «Adresse aux Honorables Chevaliers, Citoyens et Bourgeois, les Communes du Royaume-Uni de la Grande-Bretagne et d'Irlande», $1^{\text {er }}$ mars 1834, La Minerve, 24 mars ou ICMH, $\mathrm{n}^{\circ} 49037$. 
Par des allusions ou des propos plus ou moins couverts, Papineau formule alors des scénarios de menace et de rupture possible du lien colonial. En janvier 1832, il déclare en Chambre:

L'honorable membre pour Sherbrooke [M. Gugy] a beaucoup parlé de séparation des colonies de la mère patrie. Nous ne voulons pas de séparation forcée, mais il nous est permis de discuter les raisons et les causes qui y conduiront, quoi qu'assurément elle ne soit pas désirable; nous pouvons dire à ceux qui nous ont donné la constitution, «vous avez cru nous donner l'Apollon du Belvédère et nous n'avons qu'un vilain Babouin» ${ }^{51}$.

Tantôt il prend la mesure de la frustration de la Chambre d'assemblée:

Nous avons à examiner, si aujourd'hui nous ne sommes pas rendus à cette époque qu'il faut que la première magistrature de l'état recouvre le respect qu'elle a perdu, et que l'honneur, la fortune, la liberté et l'existence du peuple soient mises [sic] en sûreté, ou se résoudre à voir tomber l'un au dernier degré, et l'autre s'emporter à des excès. Oui, je le crois, nous en sommes venus à ce jour ${ }^{52}$.

Ou bien, il fait allusion à la colonie qui pourrait devenir «alliée, si la suite des tems amenait des relations nouvelles» ou alors il sert un avertissement: on doit «faire en sorte qu'on ne puisse en opprimant le Peuple de cette colonie, lui faire regretter sa dépendance de l'Empire Britannique, et chercher ailleurs remède à ses $\operatorname{maux}^{53}{ }^{3}$. Lorsque les réformes tardent encore à venir, l'impatience un tantinet menaçante se manifeste: «nous espérons, mais avec crainte, que le Gouvernement Britannique nous rendra enfin justice. Dans cet espoir nous ne ferons rien pour hâter notre séparation de la mère patrie, si ce n'est préparer et avancer le peuple pour cette époque, qui ne sera ni monarchique ni aristocratique $^{54} . »$ Puis le ton monte: il faut «comprendre que les réformes voulues par les peuples de l'Amérique Continentale, leur doivent être gracieusement octroyées, si on ne veut pas qu'un peu plus tard, elles soient forcément arrachées ${ }^{55}$ ».

Ces aveux d'impatience sont toutefois toujours tempérés par l'espoir et des attentes à l'égard de Londres. Encore après l'émeute du 21 mai 1832, Papineau reconnaît que «nous ne pouvons attendre de

51. LJP, note 16, La Minerve, 2, 9, 13 février 1832.

52. LJP, note 19, La Minerve, 27 février et 3 mars 1834.

53. LJP, note 50 , deuxième référence.

54. LJP, note 11 .

55. LJP, note 43 , dernière référence. 
redressement que du gouvernement de Sa Majesté en Angleterre ${ }^{56}{ }_{\text {». }}$. Même si elles dénoncent le Conseil législatif et réfèrent souvent aux États-Unis, les 92 Résolutions de février 1834 réitèrent la fidélité des Canadiens à l'Angleterre et leur espoir dans les droits anglais, quelle que soit la part de rhétorique de ces résolutions. Et même après avoir écrit à Arthur Roebuck en mars 1836 que la séparation morale d'avec la Grande-Bretagne était faite, Papineau affirme, au lendemain des résolutions Russell, qu'il espère encore des luttes constitutionnelles mais qu'il n'attend plus justice de la métropole. Cette prise de position paraît au mieux énigmatique, au pire contradictoire et, en un sens, typique des paradoxes de la situation politique et des procédés rhétoriques auxquels est confronté Papineau ${ }^{57}$.

La lenteur de la réponse de Londres aux 92 Résolutions et le peu de crédibilité accordée à la commission Gosford - dont on attend peu - font passer Papineau de l'impatience à la désillusion en 1836. Après avoir constaté que «la déception a été le trait saillant de chaque administration» depuis 40 ans et que les pétitions surchargent «les greniers du bureau colonial», il précise «être en lutte contre le ministre, contre le système colonial» et que les empiètements de Londres contre la Chambre d'assemblée ne peuvent que «faire naître des sentimens ruineux pour les intérêts de la métropole sur ce continent ${ }^{58}$ ». Dans son premier discours public après les résolutions Russell, le chef du Parti patriote déclare avec une certaine solennité: «La circonstance nouvelle [...], c'est que le parlement britannique prend parti contre nous.» C'était la fin de quelque chose: à la désillusion déjà vieille que les réformes puissent venir de la colonie s'ajoutait la désillusion nouvelle qu'elles puissent venir de la métropole. C'était le cul-de-sac. Papineau, qui ajoutait: «Les temps d'épreuve sont arrivés», mesurait l'impasse à laquelle était arrivée la colonie ${ }^{59}$.

En juillet 1837, les effets du cul-de-sac constitutionnel sont perceptibles. Après avoir affirmé que «le temps des pétitions» était révolu, Papineau déclare à l'assemblée populaire de L'Assomption et de Lachenaie: «nous devons espérer qu'on ne nous poussera pas aux dernières extrémités»; puis il poursuit: «Nos griefs deviennent de plus en plus intolérables» et «si malheureusement la justice reste sourde à [nos] appels, il faut que nous soyons tôt ou tard prêts à prendre ce que

56. LJP, note 46; voir aussi LJP, «PPBC: Dépêche de lord Goderich, 28 novembre 1831», La Minerve, 5 décembre 1831; LJP, note 25, deuxième référence.

57. LJP à Arthur Roebuck, 13 mars 1836, Archives nationales du Canada, Papiers Roebuck, I: 82; note 22.

58. LJP, «PPBC: Conclusion des débats de lundi dernier, 15 février 1836», La Minerve, 29 février 1836; LJP, note 41 , deuxième référence; LJP, note 21 , deuxième référence.

59. LJP, note 20 , deuxième référence. 
la main de fer du pouvoir voudrait nous arracher ${ }^{60} . »$ Conscient que lord Gosford augmente la garnison, Papineau déclare en Chambre en août 1837: «La même loi qui donne au peuple des représentans, sans le consentement desquels, on ne peut lui demander son argent, lui donne non seulement le droit, mais lui impose l'obligation de s'armer contre les rois, et les hommes des rois qui tenteraient de lui ravir le système représentatif ${ }^{61} . »$ En septembre 1837, en réponse à l'adresse de la London Workingmen's Association, Papineau et les membres du Comité central et permanent du district de Montréal écrivent: «Nous n'avons pas évoqué l'indépendance à l'endroit de la couronne britannique, mais nous n'oublions pas que la destinée des colonies continentales est de se séparer de l'état métropolitain lorsque l'action inconstitutionnelle d'un pouvoir législatif résidant en un pays lointain n'est plus supportable ${ }^{62} . »$ Voilà, immédiatement avant les rébellions de novembre 1837, l'énoncé public le plus avancé chez Papineau d'un projet de rupture avec l'Angleterre.

Lorsqu'il sera en exil en France et qu'il entreprendra (1839) de faire l'histoire de l'insurrection bas-canadienne, Papineau haussera le ton de son anticolonialisme, rappelant «comment était lourd le joug, et humiliante la condition de notre servage colonial». Il évoquera les «plaisirs Spartiates» de l'aristocratie anglaise, «celui, par exemple, de donner la chasse aux ilôtes de l'Irlande, aux ilôtes des Canadas, aux ilôtes de la Jamaïque, aux ilôtes de toutes ses possessions extérieures, toutes les fois que les serfs qui les habitent veulent cesser d'être corvéables, taillables, mortaillables à merci et miséricorde». Tout en rendant le gouvernement anglais, en métropole et en colonie, responsable des événements, Papineau n'avoue aucun repentir: «Seulement, il y a dans l'âme de tous un chagrin profond que cette résistance ait été malheureuse, mais en même temps un grand espoir qu'elle sera reprise et prévaudra ${ }^{63}$.»

\section{LE LEXIQUE NATIONALITAIRE DE PAPINEAU}

Compte tenu de cette absence de référence aux sociétés qui s'affranchissent de leur situation coloniale ou dominée, il est logique d'observer chez Papineau un lexique nationalitaire minimal, à l'exception des deux occurrences du «tyran» et de la «tyrannie». Le chef

60. LJP, note 20 , dernière référence.

61. LJP, note 30 , première référence.

62. LJP et autres signataires, "[Réponse du Comité central et permanent de Montréal à l'adresse de la London Workingmen's Association, sans date]», La Minerve, 7 septembre 1837.

63. LJP, Histoire de l'insurrection du Canada (Paris, mai 1839) [extrait de la Revue du progrès], repris à Burlington dans la Revue canadienne de Ludger Duvernay, juin 1839 et à Réédition-Québec (Montréal, 1968), 5, 11, 10. 
«Patriote» est explicite: «La patrie! La patrie! Ce mot seul suffit» comme «serment de fidélité à son pays natal». En d'autres occasions, il avouera: «L'amour du pays natal ou adoptif est le premier des devoirs, la plus belle des vertus du citoyen»; ou encore: le contrôle des subsides est «le boulevard de la liberté nationale». À la commission Gosford, il clamera: «nous représentons un peuple ${ }^{64}$.» Voilà. Pas de récurrence de termes comme «indépendance», «souveraineté», «nation» ou «national», «libération» ou la constellation de leurs synonymes possibles. Des occurrences certes, mais point de récurrence. Et même dans le cas d'usage du vocable «national», par exemple, la signification à donner au terme est loin d'être évidente. Le lexique dominant, encore que pas très fréquent, est celui de «patrie» ou de «Patriotes» (individu, parti). Le patriotisme apparaît donc comme l'espace sémantique dans lequel évolue Papineau, comme la définition de sa «fidélité à son pays natal».

Il a certes une représentation du «pays», du «sol natal» et des composantes usuelles d'un peuple et de la nation. Papineau connaît l'histoire du Canada et l'histoire parlementaire du Bas-Canada; il en est l'artisan et le témoin, il y réfère constamment, il en favorise l'histoire écrite, expliquant même que si la Chambre a financièrement aidé à la publication des cartes géographiques de Bouchette, elle doit manifestement venir en aide à la publication du manuscrit de l'Histoire du Canada du docteur Jacques Labrie ${ }^{65}$. Sa conscience historique inclut aussi la connaissance de l'histoire de la Grande-Bretagne et de ses colonies et de celle des États-Unis.

Papineau témoigne aussi de son attachement au sol: par le maintien du régime seigneurial, qui ne lui paraît pas fonder une aristocratie canadienne locale, alors qu'il décrie l'aristocratie anglaise coloniale avec vigueur et constance et à laquelle il s'oppose en «démocrate»; par l'attention portée à l'exploitation du domaine public et aux modes de distribution des terres des Cantons en particulier. Papineau tient ses propos les plus radicaux lorsqu'il s'agit de dénoncer la British

64. LJP, «À nos Constituans», 2 avril 1827, La Minerve, 26 mars 1827; LJP, note 15; notes $19,35,48$ et 41 , deuxième référence.

65. LJP, «PPBC: Cas du Col. Bouchette, 19 févier 1827», La Minerve, 26 février; «PPBC: Pétition de Mad. Labrie, 30 novembre 1831», Le Canadien, 3 décembre 1831; «PPBC: Histoire ancienne du pays, 12 décembre 1831», La Minerve, 22 décembre 1831; «PPBC: Arts et sciences, 13 novembre 1835», La Minerve, 19 novembre 1835. 
American Land Company ou l'usage fait, pour des motifs de patronage, des biens des jésuites ${ }^{66}$.

Son attachement à la langue française est d'abord et avant tout visible dans le combat qu'il mène pour le perfectionnement du système judiciaire en général et pour la défense du français parlé dans les cours de justice en particulier. Tout comme Parent, il soutient que c'est la langue française et la différence culturelle qui ont sauvé la colonie en 1774 et qu'il n'est «pas nécessaire de cesser de parler le français pour connaître et chérir la constitution». Le système juridique distinct du Bas-Canada sert d'ailleurs d'argument à Papineau en 1823, pour contrer le projet d'Union de $1822^{67}$.

Quant à la religion dont il parle peu, sinon pour affirmer la dimension privée de la croyance, la nécessité de la tolérance religieuse (égalité de toutes les dominations religieuses face aux réserves du clergé) et l'imputabilité temporelle de l'Église (question des notables, aide gouvernementale aux religieuses hospitalières), elle est le rappel que sur cette question particulière, Papineau a su taire ses convictions profondes de déiste et se limiter à des combats publics susceptibles d'être partagés par d'autres libéraux ${ }^{68}$.

Si le lexique nationalitaire de Papineau est minimal, c'est que le chef du Parti patriote est le pourfendeur des «distinctions nationales». Idéologiquement et politiquement, Papineau ne peut et ne veut attiser les différences nationales. Il considère que les «antipathies nationales» constituent «un sentiment dégradant quand on a voyagé». Les occasions sont fréquentes où Papineau récuse les «distinctions nationales»: l'université qu'il envisage à partir de la restitution des biens des jésuites sera «sans aucune distinction», le choix d'un agent de la Chambre d'assemblée à Londres doit se faire indépendamment du fait qu'il soit né ou pas au Bas-Canada, sa propre candidature électorale doit avoir «le support de ceux, qui sans distinction, se vantaient d'appartenir à la

66. LJP, «PPBC:[Adresse à Son Excellence George Comte de Dalhousie], 22 décembre 1820», Le Canadien, 27 décembre 1820; LJP, «PPBC: Réélection des employés publics, 8 février 1831», La Minerve, 14 février 1831; LJP, «PPBC: Réponse à la Harangue du gouverneur, [s.d.]», Le Canadien, 9 mars 1835; LJP, «PPBC: Bill pour la régie des biens des Jésuites, 30 janvier 1836», Le Canadien, 3 février 1836; LJP, «PPBC: Second rapport du comité des griefs, 15 février 1836», La Minerve, 25 février 1836; LJP, note 20, troisième référence.

67. LJP, «PPBC: Message du gouverneur relatif à la composition du grand jury pour la cour criminelle de Montréal, 6 mars 1833», La Minerve, 28 mars 1833; LJP, note 7; LJP, note 4 , deuxième référence.

68. LJP, note 36 , première référence; LJP, note 42 , première référence, La Minerve, 27 septembre 1827; LJP, «PPBC:Hôtel-Dieu de Québec, 12 janvier 1829», La Minerve, 22 janvier 1829; LJP, «PPBC: [Sur les amendements du Conseil au bill des congrégations religieuses], 8 mars 1836», Le Canadien, $1^{\text {er }}$ avril 1836; LJP, «PPBC: Hôpital des émigrés, 24 février 1834», La Minerve, 20 mars 1834. 
classe des réformateurs libéraux sans examen de pays», les dénominations religieuses doivent sans distinction avoir les mêmes droits; il regrette par ailleurs «les vues exclusives et les préjugés nationaux dont [la Quebec Literary and Historical Society] a fait preuve dès son origine ${ }^{69}$ ». L'orateur de la Chambre reconnaît qu'il «méprisait autant ceux de son origine qui trahissaient la confiance publique, qu'il respectait ceux d'origine différente qui agissaient honnêtement». À la presse anglophone ou aux Adam Thom qui reviennent sans cesse sur les préjugés nationaux, Papineau répond:

On feint de croire que nos réclamations sont le fruit de notre différence d'origine et du catholicisme, quand il est constant que les rangs des libéraux comptent une majorité des hommes de toute croyance et de toute origine. Mais que dire à l'appui de cet avancé, quand on voit le Haut-Canada où il n'y a que peu de catholiques et où presque tous les habitans sont d'origine anglaise, dénoncer les mêmes maux et demander les mêmes réformes $^{0}$ ?

Tout en se défendant de susciter ou d'entretenir ces «distinctions nationales», Papineau montre du doigt les institutions responsables de la création et du maintien des «distinctions nationales»: c'est le système de nomination aux places, c'est le système des réserves du clergé qui avantage le clergé anglican et presbytérien - le clergé catholique romain a des seigneuries - mais c'est surtout le Conseil législatif qui, au fil des années et des nominations, s'est donné un rôle inacceptable:

sa prétention injuste à n'avoir pour mission que de donner de la sécurité à une classe particulière des Sujets de Sa Majesté en cette Province, comme ayant des intérêts qui ne pourraient être suffisamment représentés dans cette Assemblée [...]. Une prétention de cette nature est une violation de la Constitution, et ne peut que susciter et perpétuer, entre les diverses classes des habitans de la Province, des méfiances, des distinctions et des animosités nationales, et tendre à donner à une partie du Peuple une supériorité injuste et factice sur l'autre, avec l'espoir de la domination et d'une préférence indue ${ }^{71}$.

69. LJP, «PPBC: Finances, 10 mars 1830», La Minerve, 18 mars 1830; LJP, «PPBC: Réélection des employés publics, 8 février 1831», La Minerve, 14 février 1831; LJP, «PPBC: Nomination d'un agent, 5 mars 1831», La Minerve, 14 mars 1831; LJP, note 33; LJP, «PPBC: Musée Chasseur, 25 février 1836», La Gazette de Québec, 12 mars 1836.

70. LJP, «Requête de la convention de Montréal», 23 février 1835, La Gazette de Québec, 24 février 1835; LJP, note 41, deuxième référence, La Minerve, 14 mars 1836.

71. LJP, note 16, Le Canadien, 4 février 1832; LJP, note 50, deuxième référence. 
Encore en août 1837, Papineau revient sur cette identification du vice par excellence de la situation coloniale sur lequel lord Durham n'insistera pas:

C'est le conseil lui-même qui [...] a révélé la bassesse de ses prédilections et de ses antipathies, s'est déclaré l'organe et l'instrument passif d'une faction, s'est voué à fomenter et protéger des distinctions nationales, comme élément à conserver et d'après lequel le gouvernement devait être constitué, comme si les maux de l'Irlande et l'opprobre de l'Angleterre, ne les avaient pas suffisamment avertis que ce principe était une inspiration de l'enfer [...]. Le Lord Gosford et ses officiers, et le Lord Glenelg sont tombés sous l'empire des mêmes puissances de ténèbres, lorsqu'ils ont accueilli l'inique distinction. L'un fonda sur cette circonstance le refus de rendre le Conseil électif; l'autre dit dans les débats que nos dissensions ne sont pas une lutte de principes, mais d'origine ${ }^{72}$.

Et pourtant, répétait Papineau, les revendications sont les mêmes dans le Haut et dans le Bas-Canada...

Si le refus des «distinctions nationales» persiste chez Papineau immédiatement après les rébellions, la détermination indépendantiste s'affermit. La conclusion qu'il faut tirer, selon lui, du Rapport de lord Durham est «que les Canadiens n'ont aucune justice à attendre de l'Angleterre; que pour eux, la soumission serait une flétrissure et un arrêt de mort, l'indépendance, au contraire, un principe de résurrection et de vie»; puis, écrivant pour un public français, il ajoute: «le rapport de lord Durham servira aussi à prouver que l'indépendance du Canada [le souligné est de nous] est un événement voulu par l'intérêt de l'ancienne comme de la nouvelle France, et par l'intérêt de l'humanité tout entière». Et l'Histoire de l'insurrection du Canada se termine ainsi:

Un récit historique, impartial et succinct, des événements qui se sont déroulés dans mon pays pendant les deux dernières années portera dans tous les esprits cette conviction que ce ne sont pas les statuts anglais qui règleront le prochain avenir du Canada; mais que cet avenir est écrit dans les déclarations des droits de l'homme et dans les constitutions politiques que se sont données nos bons, sages et heureux voisins, les Américains indépendants $^{73}$.

72. LJP, note 30, première référence, Le Libéral, 5 septembre 1837.

73. LJP, Histoire de l'insurrection du Canada, mai 1837, op. cit., 13-14, 34. 


\section{CONCLUSION}

Comment la conscience coloniale de Papineau s'arrime-t-elle donc à sa conscience internationale? La conscience coloniale de Papineau est au départ une conscience revendicative tempérée par une admiration des institutions et de la constitution britanniques. En cela, Papineau appartient à une tradition politique familiale et canadiennefrançaise. Non seulement s'inscrit-il d'abord dans cet héritage, mais encore contribue-t-il à la transformation de cette tradition de britannisme. Il est d'abord admirateur de la constitution de 1791 parce que, fondamentalement, le parlementarisme bas-canadien peut se fonder sur le principe représentatif, sur la souveraineté populaire. Sur ce fondement, sa pensée et son action politiques visent à tirer toutes les implications du statut de «sujet anglais» et à exiger tous les droits des sujets - et pas des citoyens - de l'Angleterre.

En cela Papineau est profondément américain: sa démarche politique et constitutionnelle ressemble de part en part à celle des penseurs des Treize colonies beaucoup plus qu'à celle des Français qui feront 1789. Comme les Américains qui recherchent d'abord, de façon légitime, leur autonomie en s'appuyant sur leurs «droits de sujets anglais», Papineau mène le combat de l'autonomie fiscale de la colonie avant d'être amené à considérer son autonomie constitutionnelle. Son défi est simple mais risqué: comment maintenir l'équilibre entre l'allégeance et l'origine, entre la loyauté britannique et la recherche puis la découverte d'une identité canadienne et bas-canadienne (langue, religion, tenure, lois)? La trame de la pensée politique de Papineau peut se résumer par cette idée que ses premières revendications prennent appui sur la qualité, sur les droits des sujets anglais alors que ses dernières se fondent sur «l'égalité naturelle des hommes au sein de l'Empire $^{74} \gg$. En ce sens, un individu peut évoluer légitimement et progressivement de la monarchie constitutionnelle à la république, de la loyauté à l'affirmation d'une identité coloniale. Et toute cette recherche d'autonomie est la résultante obligée, au XVIII ${ }^{\mathrm{e}}$ et au $\mathrm{XIX}^{\mathrm{e}}$ siècles, de l'octroi aux colonies de la souveraineté populaire par les métropoles, à cette particularité près que la souveraineté dans la colonie ne peut que donner le goût de la souveraineté de la métropole.

L'admiration de Papineau à l'égard de l'Angleterre est ébranlée à la fin des années 1820 par son insatisfaction et celle du Parti canadien

74. Richard Larue, «Allégeance et origine: contribution à l'analyse de la crise politique au Bas-Canada», Revue d'histoire de l'Amérique française, 44,4 (printemps 1991): 547-548; Élise Marienstras, Nous, le peuple. Les origines du nationalisme américain (Paris, Gallimard, 1988), en particulier le chapitre IX, «La nature de l'Empire et l'identité politique des colonies», 163-179. 
à propos du statut non électif et de la composition non représentative du Conseil législatif. Le blocage constitutionnel colonial - le Conseil législatif refusant de voter les lois passées par la Chambre d'assemblée - crée alors un ressort fondamental de la pensée et de la stratégie de Papineau. À ce propos, les attentes de solutions au problème colonial sont dorénavant directement proportionnelles aux désillusions qui viennent avec les accommodements proposés dans la colonie par le gouverneur et son Exécutif. La métropole est alors investie de pouvoirs et d'espoirs qui tempèrent le style revendicatif de Papineau et du Parti patriote. Mais il faut traverser le désert des doléances, des pétitions, des représentations, du côté colonial, et celui des intérêts des ministres successifs des colonies et de l'Empire par excellence du $\mathrm{XIX}^{\mathrm{e}}$ siècle, du côté métropolitain, avant de pouvoir constater le culde-sac de la stratégie de la patience et avant de devoir envisager autre chose. Telles sont les composantes essentielles de la conscience coloniale de Papineau.

La recherche d'exemples ou d'appuis n'incite pas Papineau à se tourner vers la France. La francophobie des anglophones de la colonie le rend vigilant face à tout signe de francophilie de sa part. De plus, 1789 et surtout 1793 sont perçus par un Papineau admirateur de 1688 et de 1776, Napoléon n'a pas bonne presse et 1830 ne paraît pas être une référence pour le chef du Parti patriote, quelles qu'en soient les raisons. Papineau peut donc protester avec sincérité d'un «attachement aux principes français» que ses adversaires lui imputent. La chose est d'autant plus concevable que son sens démocratique et républicain se mesure aux meilleures aunes: celle de l'Angleterre et surtout celle des colonies qui, elles, ont fait leur indépendance, les États-Unis.

C'est ici qu'apparaît le génie discret de la pensée et de la rhétorique de Papineau: rester dans la mouvance britannique en réclamant tous les droits du «sujet anglais», en référant au Royaume-Uni et aux autres colonies britanniques et en focalisant sur l'expérience réussie des États-Unis plutôt que de provoquer la métropole en référant à 1789 , à 1793, à la Grèce, à l'Italie ou à la Pologne. Cette approche est délibérée; en effet, comparée aux références faites par Étienne Parent ou François-Xavier Garneau à la Pologne, par exemple, ou aux références de la presse francophone en général aux mouvements d'émancipation en Europe et en Amérique latine, cette stratégie qui fait silence sur cette réalité évoquée tout autour de Papineau ne peut-être que voulue ${ }^{75}$. Il faut de plus, pour comprendre le tonus revendicatif de

75. Y. Lamonde, «Une 'soumission honorable': Étienne Parent, la situation internationale et l'émancipation coloniale (1822-1840)» (à paraître); “'L'ombre du passé': François-Xavier Garneau et l'éveil des nationalités», Actes du colloque Garneau (à paraître); recherche en cours 
Papineau et les composantes de sa conscience internationale, se rappeler que sa radicalisation politique (élection du 22 mai 1832, 92 Résolutions de février 1834) est légèrement postérieure aux événements qui marquent la France, la Belgique et la Pologne en 18301831.

On peut faire une autre analyse de cette non-référence chez Papineau à des pays qui ont expressément voulu ou obtenu leur indépendance. En effet, sauf pour les États-Unis qui ont fait leur indépendance, la référence à l'Irlande et à la Jamaïque indiquerait-elle que Papineau, avant 1839, ne revendique qu'une certaine autonomie coloniale, qu'une émancipation partielle? Par contre, si tel était le cas, comment comprendre que la demande du gouvernement responsable, mesure plus réformiste, occupe si peu de place dans son discours, comparativement aux questions de la liste civile et de l'électivité du Conseil législatif qui paraissent alors plus radicales ${ }^{76}$ ?

La diagonale de la conscience internationale de Papineau passe quelque part entre l'indépendance obtenue et faite par les États-Unis et les réformes obtenues et encore à obtenir par l'Irlande. De ce point de vue, la figure de O'Connell est garante des réformes demandées par le Bas-Canada, exploité de la même façon que l'Irlande, exigeant des réformes identiques et devant comme l'Irlande se faire craindre.

La place des États-Unis dans le discours de Papineau s'explique ainsi comme un rappel, comme un coin fiché dans la mémoire métropolitaine. Le processus n'est pas violent, mais il a son efficacité quand on pense à l'attention que le secrétariat d'État aux Colonies et le Rapport de lord Durham portent aux États-Unis dans l'analyse de la dynamique coloniale nord-américaine. Papineau ne se sert pas des États-Unis uniquement pour marquer le terrain des revendications et baliser les étapes de ses projets - électivité du Conseil législatif, nonconsommation de produits importés - ce qui est déjà beaucoup, mais aussi pour constituer une identité américaine aux Canadiens et aux Canadiens français, pour façonner leur appartenance sinon à l'hémisphère américaine - la référence à l'Amérique latine est très peu fréquente - du moins à l'Amérique du Nord. Les Canadiens et les Canadiens français ne sont ni anglais, ni français, ni européens; ils sont canadiens et canadiens-français d'Amérique, du Nouveau Monde. Si la pensée de Papineau à ce sujet ne permet pas de parler de «doctrine Papineau» comme on parle de «doctrine Monroe», il n'en reste

\footnotetext{
sur les mouvements nationalitaires européens et américains dans la presse francophone bascanadienne (1808-1838).

76. F. Murray Greenwood, «Les patriotes et le gouvernement responsable dans les années 1830», Revue d'histoire de l'Amérique française, 33,1 (juin 1979): 25-37.
} 
pas moins que cette insistance sur la dimension américaine est en soi une distance culturelle et politique de la métropole. Lorsque Papineau affirme que le Bas-Canada ne fait que demander ce qui a été accordé aux «anciennes colonies», il escompte pouvoir obtenir, un demi-siècle plus tard, par des luttes constitutionnelles, ce que les États-Unis ont dû obtenir par une guerre d'indépendance. Mais il a aussi vraisemblablement à l'esprit le fait que, déjà avant l'Indépendance, des colonies comme le New Hampshire et le Connecticut disposaient d'un gouverneur et d'un Conseil élus. À un dernier titre, la référence étatsunienne est éloquente à propos de la pensée internationale de Papineau: elle explique son annexionnisme de 1849 en faisant voir que s'il n'est pas favorable à l'intégration aux États-Unis avant 1837 il a, alors, une représentation de «l'indépendance souveraine» des États constituant les États-Unis. Mais comment obtenir ce type de «souveraineté indépendante» en dehors de l'Angleterre et en dehors des États-Unis? Et si c'est pour l'être sous pavillon anglais ou étatsunien, que peut vouloir dire «souveraineté indépendante»? Papineau ira plus loin dans son Histoire de l'insurrection du Canada de 1839, mais cette position est autre chose.

Il y a toutefois dans cette rhétorique de fil de fer quelques prouesses périlleuses, une évidente radicalisation balisée par des dates repères: le projet d'Union de 1822, l'émeute électorale du 21 mai 1832, les 92 Résolutions de février 1834 , le peu de crédibilité des travaux de la commission Gosford de 1835 et la massue des résolutions Russell de mars 1837. Mais cette radicalisation est bien tempérée. Le ton monte puis se pose: Papineau refuse la «séparation forcée», mais il prévient qu'on ne doit pas «faire regretter la dépendance à l'Empire britannique» ou que des réformes non «octroyées» pourraient être «arrachées». Encore après les résolutions Russell, il défend l'approche par les luttes constitutionnelles mais affirme ne plus attendre justice de la métropole.

Après le refus de Londres en mars 1837, sa conscience de la condition coloniale est confrontée à un cul-de-sac, obligeant ainsi le chef du Parti patriote à évoquer «la destinée des colonies continentales» ou à rappeler au peuple «l'obligation de s'armer contre les rois, et les hommes des rois qui tenteraient de lui ravir le système représentatif», et ce, au moment où les autorités coloniales renforcent les garnisons. Outre ce rappel de principe, rien cependant dans les interventions de Papineau ne peut constituer un appel aux armes. Dix ans de tensions coloniales, dix ans de tergiversations métropolitaines, dix ans d'attentes finalement déçues, la pression militaire britannique, la radicalité de certains éléments du Parti patriote ajoutée à d'autres 
causes permettent de comprendre que la situation ait évolué vers un goulot d'étranglement dont la responsabilité est difficilement imputable à une personne ou à un seul des protagonistes.

Avant 1837, Papineau ne paraît donc pas faire appel explicitement au principe des nationalités, au droit des peuples à disposer d'euxmêmes. La formule qui s'en rapproche le plus avant 1837 est celle de «la destinée des colonies continentales». Après 1837, la référence est plus explicite lorsqu'il parle de «l'indépendance» comme «principe de résurrection et de vie» ou d'un avenir du Canada «écrit dans les déclarations des droits de l'homme et dans les constitutions politiques que se sont données nos bons, sages et heureux voisins, les Américains indépendants». Papineau est alors tout à fait logique avec sa pensée et sa démarche: tout en restant dans l'univers britannique, il garde son regard braqué sur l'expérience des colonies qui avaient fait de leurs «sujets» des citoyens. 